\title{
Beam commissioning for a superconducting proton linac
}

Zhi-Jun Wang, ${ }^{*}$ Yuan He, ${ }^{\dagger}$ Huan Jia, Wei-ping Dou, Wei-long Chen, X. L. Zhang, ${ }^{*}$ Shu-hui Liu, Chi Feng, Yue Tao, ${ }^{\S}$ Wang-sheng Wang, Jian-qiang Wu, Sheng-hu Zhang, and Hong-Wei Zhao Institute of Modern Physics, Chinese Academy of Sciences, Lanzhou 73000, China

(Received 26 January 2016; published 27 December 2016)

To develop the next generation of safe and cleaner nuclear energy, the accelerator-driven subcritical (ADS) system emerges as one of the most attractive technologies. It will be able to transmute the longlived transuranic radionuclides produced in the reactors of today's nuclear power plants into shorterlived ones, and also it will provide positive energy output at the same time. The prototype of the Chinese ADS (C-ADS) proton accelerator comprises two injectors and a $1.5 \mathrm{GeV}, 10 \mathrm{~mA}$ continuous wave $(\mathrm{CW})$ superconducting main linac. The injector scheme II at the C-ADS demo facility inside the Institute of Modern Physics is a $10 \mathrm{MeV} \mathrm{CW}$ superconducting linac with a designed beam current of $10 \mathrm{~mA}$, which includes an ECR ion source, a low-energy beam transport line, a $162.5 \mathrm{MHz}$ radio frequency quadrupole accelerator, a medium-energy beam transport line, and a superconducting half wave resonator accelerator section. This demo facility has been successfully operating with an $11 \mathrm{~mA}$, 2.7 MeV CW beam and a $3.9 \mathrm{~mA}, 4.3 \mathrm{MeV} \mathrm{CW}$ beam at different times and conditions since June 2014. The beam power has reached $28 \mathrm{~kW}$, which is the highest record for the same type of linear accelerators. In this paper, the parameters of the test injector II and the progress of the beam commissioning are reported.

DOI: 10.1103/PhysRevAccelBeams.19.120101

\section{INTRODUCTION}

The accelerator-driven subcritical (ADS) system, one of the technologies for a clean nuclear power source, was proposed a long time ago [1-3]. To satisfy the fast-growing needs of the country's economic development and combat global warming, the next generation of safer and cleaner nuclear energy is needed. The ADS system emerges as one of the most attractive cutting-edge technologies to transmute minor actinides and long-lived fission products produced in the reactors of today's nuclear power plants (as nuclear waste) into relatively much shorter-lived or stable ones to largely reduce the burden of nuclear waste management and at the same time to generate positive energy output. It is reckoned as one of the vital measures at the forefront of the exploration for sustainable energy production. Therefore, the Chinese ADS (C-ADS) proofing project was launched in 2011 under the management of the Chinese Academy of Sciences [4,5]. The design of the ADS proton accelerator comprises an injector linac and a

\footnotetext{
*wangzj@impcas.ac.cn

hey@impcas.ac.cn

Guest scientist.

${ }^{\S}$ Also at University of the Chinese Academy of Sciences, Beijing 100049, China.
}

Published by the American Physical Society under the terms of the Creative Commons Attribution 3.0 License. Further distribution of this work must maintain attribution to the author(s) and the published article's title, journal citation, and DOI.
$1.5 \mathrm{GeV}, 10 \mathrm{~mA}$ continuous wave $(\mathrm{CW})$ superconducting main linac.

The accelerator is developed by collaborations between Institute of Modern Physics (IMP) and Institute of High Energy Physics. The road map and the layout of the C-ADS project are shown in Fig. 1. The demonstrative project was divided into three stages. Stage one: A research facility including a $250 \mathrm{MeV}, 10 \mathrm{~mA} \mathrm{CW}$ superconducting proton linac with a test reactor will be built with a reactor power of 10 MW by the year 2022. At this stage, many critical technologies will be tested and the principle system will be synthesized. Stage two: Most of the challenges will hopefully be resolved, and the energy of the linac will be increased to $1.0 \mathrm{GeV}, 10 \mathrm{~mA}$ with a reactor power of $100 \mathrm{MW}$ for an operational demonstration ADS system. Stage three: A $1.5 \mathrm{GeV} \mathrm{CW}$ accelerator with a beam current of 10-25 mA plus a $1 \mathrm{GW}$ reactor commercial prototype system will be built for performance studies of the daily operation.

The injector scheme II being built at IMP is composed of an ion source, a low-energy beam transport line (LEBT), a $162.5 \mathrm{MHz}$ radio frequency quadrupole accelerator (RFQ), a medium-energy beam transport line (MEBT), and a superconducting half wave resonator (HWR) accelerator section [5-7]. The machine design and simulation is mainly carried out by using the dynamics simulation codes of TraceWin [8], TRACK [9], PARMTEQ (for RFQ) [10], and BEAMPATH (mainly for LEBT) [11]. These codes have been used in many projects. The layout of the injector scheme II is shown in Fig. 2, and the beam dynamics simulation results are shown in Fig. 3, where the beam rms envelopes 

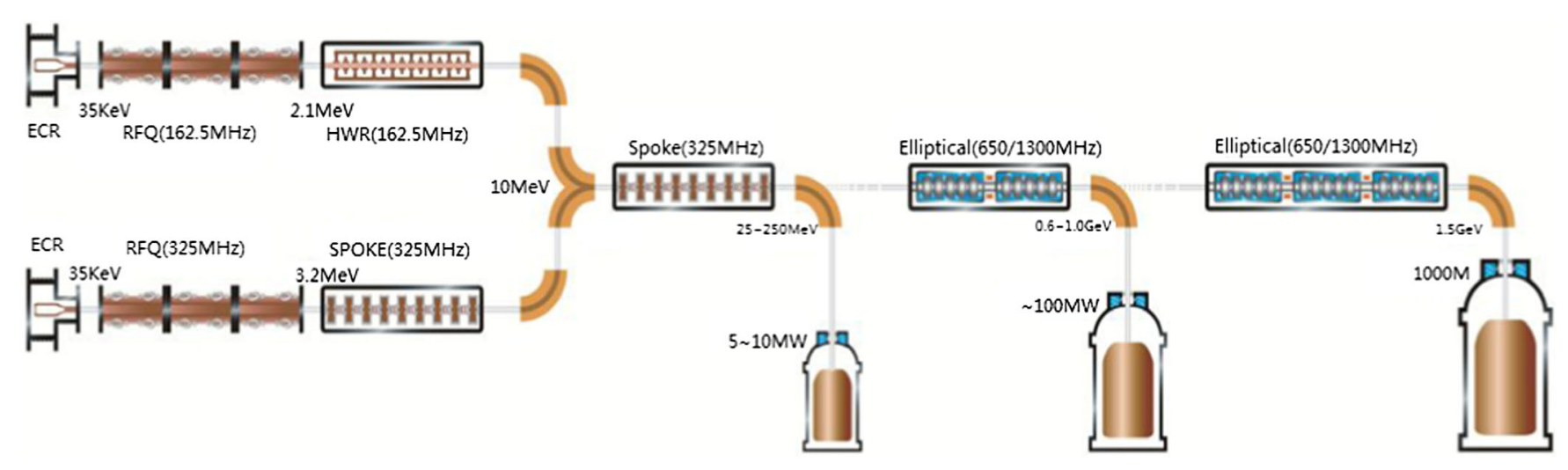

FIG. 1. Layout of the C-ADS.

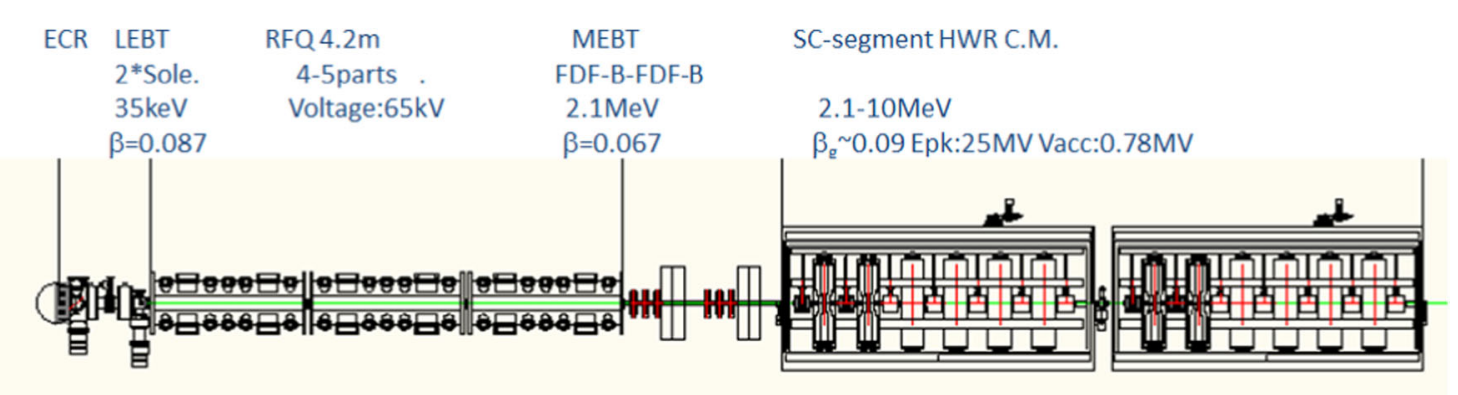

FIG. 2. Layout of the C-ADS injector scheme II.
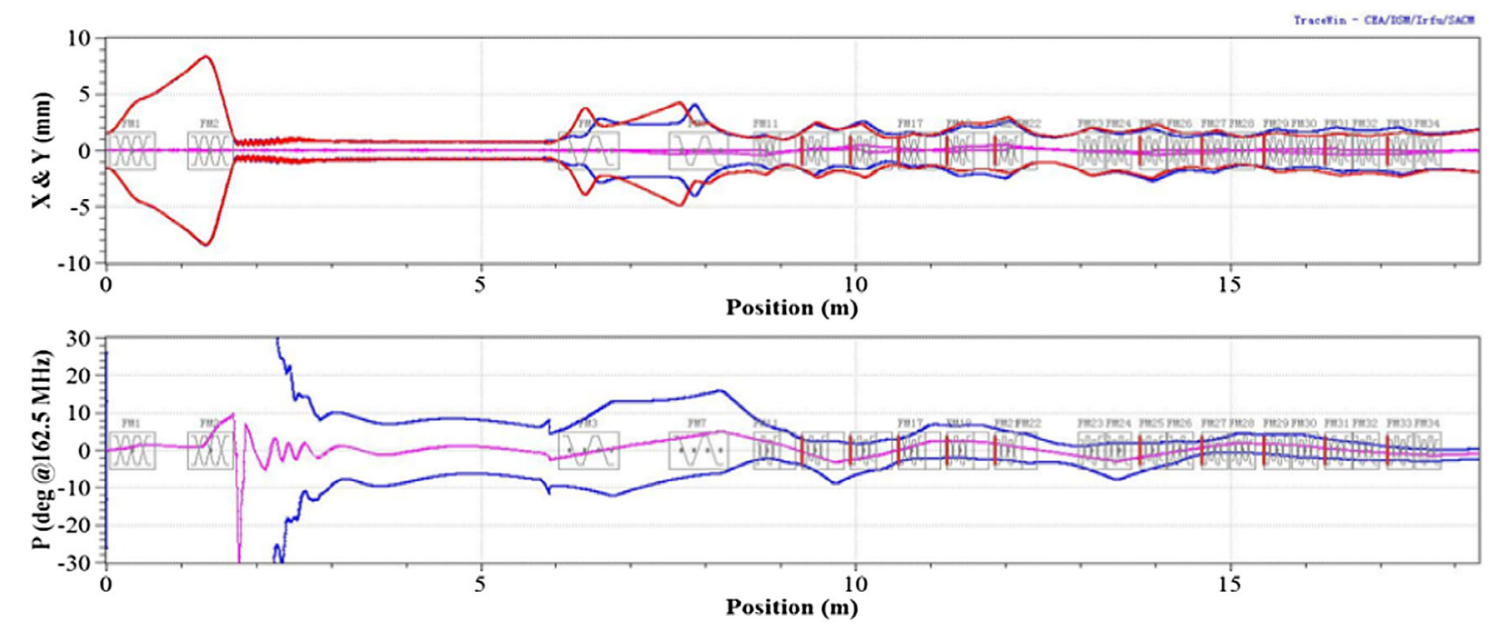

FIG. 3. Beam physics design of the C-ADS injector scheme II.

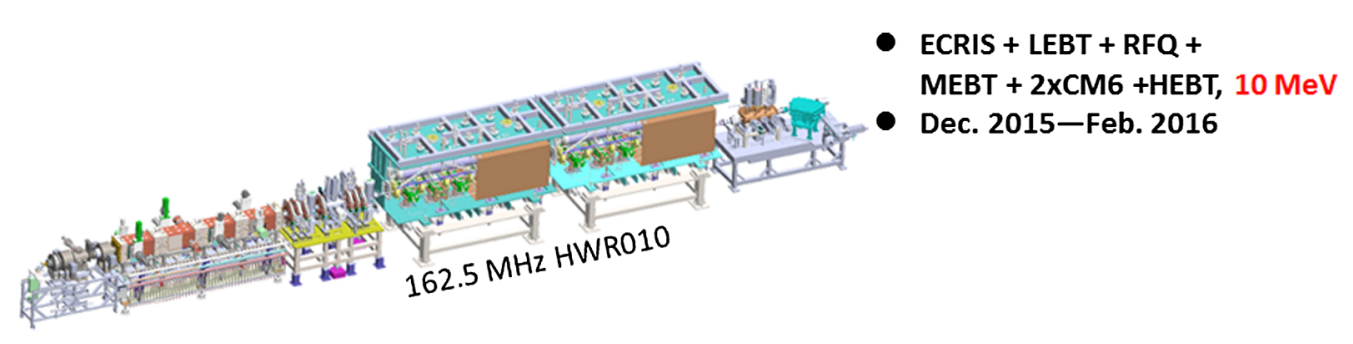

FIG. 4. Mechanic drawing of the injector scheme II. 
TABLE I. The main parameters of injector scheme II.

\begin{tabular}{llc}
\hline \hline Energy & $E(\mathrm{MeV})$ & 10 \\
Length & $L(\mathrm{~m})$ & 19 \\
Radio frequency & $f(\mathrm{MHz})$ & 162.5 \\
Emittance $(\mathrm{rms})$ & $\epsilon_{x}(\pi \mathrm{mm} \mathrm{mrad})$ & 0.2 \\
Current & $I(\mathrm{~mA})$ & 10 \\
\hline \hline
\end{tabular}

in both the transverse and longitudinal directions are plotted. (In this paper, the beam emittance and envelopes are all $\mathrm{rms}$ values if not especially mentioned.)

Since it is the first superconducting $\mathrm{CW}$ proton linac in China, the designs and operations of similar linacs all over the world have been studied. Especially, the experiences of the Spallation Neutron Source [12] and the Soreq Applied Research Accelerator Facility have been carefully studied [13]. The strategy is set as the beam commissioning continues as each subsystem installation finishes [14]. In the following sections, the details of the machine commissioning studies of the injector scheme II will be presented.

\section{COMMISSIONING AND THE BEAM STUDIES FOR THE INJECTOR SCHEME II}

For the injector scheme II shown in the introduction, the mechanic installation layout is shown in Fig. 4. The main parameters are listed in Table I. It has an ECR proton source, a LEBT, a $162.5 \mathrm{MHz}$ RFQ cavity, a MEBT, and superconducting HWR accelerating cavities.

The accelerator control system is based on EPICS and CSS and some with Labview applications for fast development. There are also a lot of programs written in Labview and Matlab for beam diagnostics. In addition, to aid the beam commissioning and measurement, there is a special movable beam instrumentation table (D plate) built and mainly used in pulse mode as is shown in Fig. 5. There are one analyzing magnet with a beam slit and Faraday cup for beam energy and energy spread measurement, three beam position monitors (BPMs) for time of flight (TOF) measurement, an ac current transformer (ACCT), and a dc current transformer (DCCT), several horizontal and vertical beam slits and scanning wires to measure the beam emittance, a fast Faraday cup (FFC) for bunch shape, and a normal Faraday cup for beam current, and a movable Faraday cup acts temporarily as a beam dump.

\section{A. The proton source and the LEBT}

The parameters and the layout of the ECR proton source and the LEBT are shown in Table II and Fig. 6.

The LEBT has two focusing solenoids with horizontal and vertical orbit correction coils [15]. There are a number of beam diagnostic instruments including a DCCT and horizontal and vertical beam slits in both test chambers. The proton source can be operating at a $1 \mathrm{~Hz}$ pulse mode or $\mathrm{CW}$ mode. For beam commissioning and measurement, the pulsed mode was usually adopted. In addition, there are a cone electrode and a beam chopper to shape the beam transversely and longitudinally.

Before the installation of the RFQ, the beam emittance was measured by the slits at the position $85 \mathrm{~mm}$ after the LEBT exit. The beam distribution in transverse phase space is shown in Fig. 7 at $15 \mathrm{~mA} \mathrm{CW}$ beam. The normalized beam emittance deduced from the measurement is about $0.13 \pi \mathrm{mm}$ mrad when we traced back to the RFQ entrance, and it satisfies the design goal. Also, the estimated space

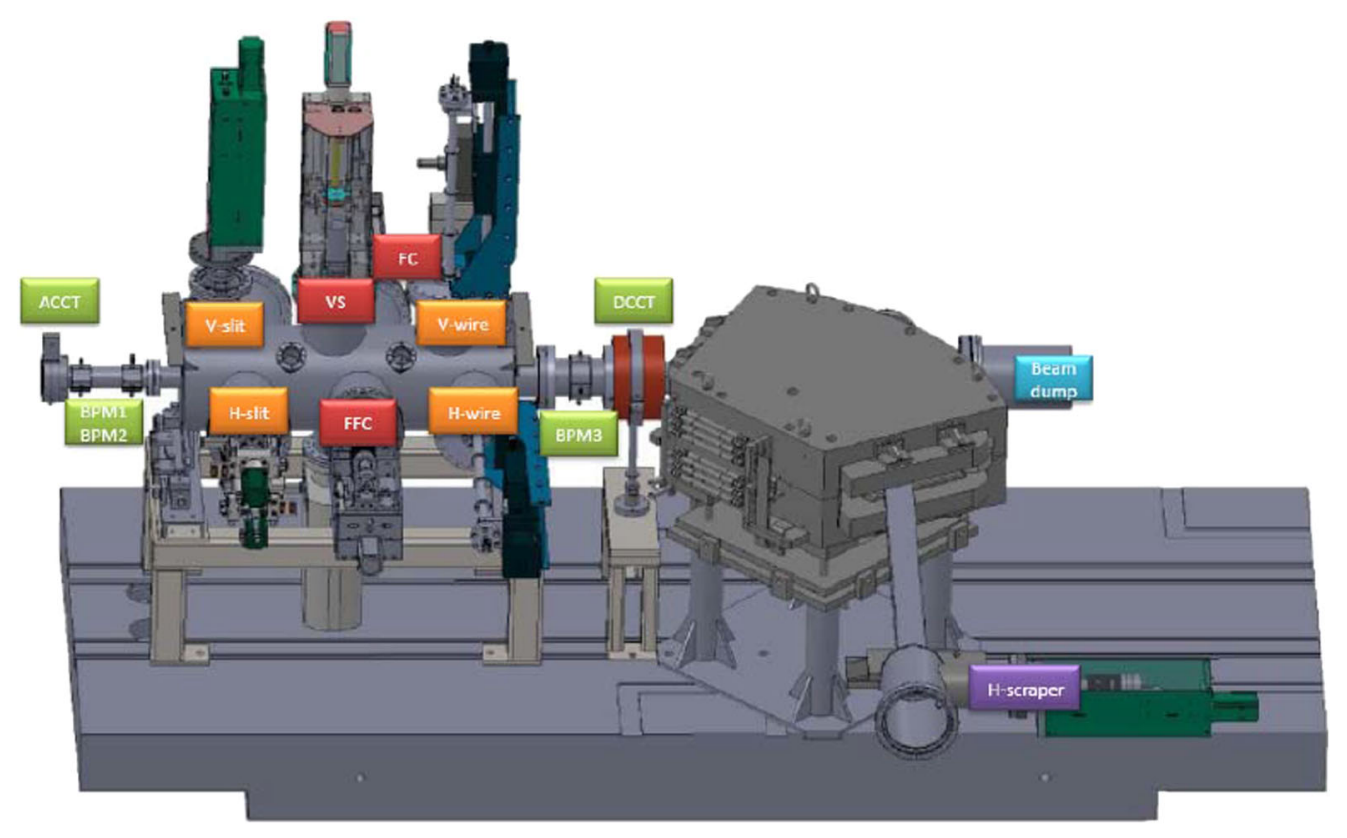

FIG. 5. Movable beam measuring platform. 
TABLE II. The main design parameters of the ECR source and LEBT.

\begin{tabular}{llc}
\hline \hline Energy & $E(\mathrm{keV})$ & 35 \\
Length & $L(\mathrm{~m})$ & 1.67 \\
Radio frequency & $f(\mathrm{GHz})$ & 2.45 \\
Emittance (rms) & $\epsilon(\pi \mathrm{mm}$ mrad $)$ & 0.2 \\
Current & $I(\mathrm{~mA})$ & 15 \\
\hline \hline
\end{tabular}

charge compensation factor is about 0.9 , which is obtained from the experiment.

\section{B. The RFQ accelerator}

The RFQ cavity was designed via the collaboration between the IMP and LBNL and fabricated at IMP. It can bunch and accelerate the proton beam from $35 \mathrm{keV}$ to 2.1 $\mathrm{MeV}[16,17]$. The main parameters are shown in Table III, and the commissioning layout of the RFQ cavity is shown in Fig. 8.

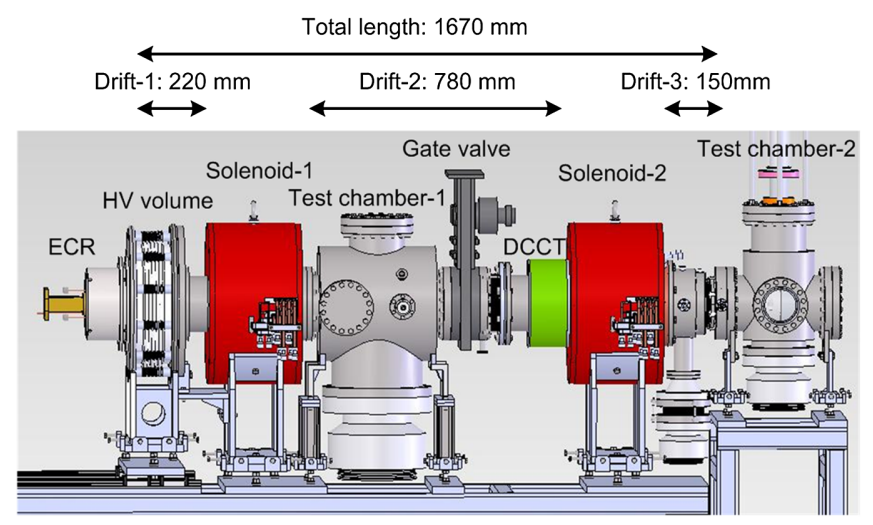

FIG. 6. Layout of the LEBT.

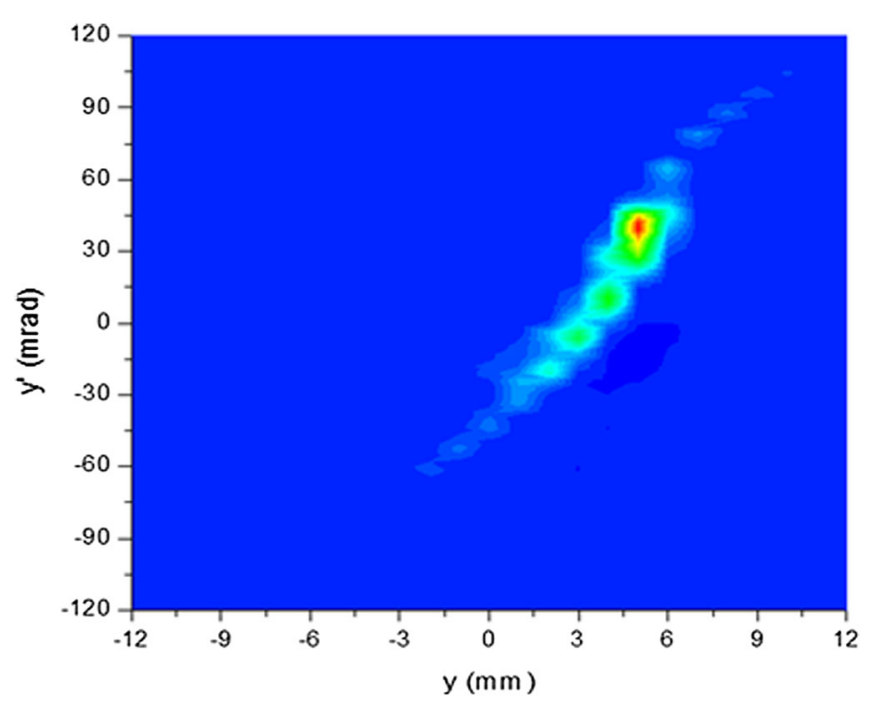

FIG. 7. Normalized vertical emittance at $85 \mathrm{~mm}$ after the LEBT exit.
TABLE III. The main design parameters of the RFQ.

\begin{tabular}{llc}
\hline \hline Energy & $E_{\text {in }} / E_{\text {out }}(\mathrm{MeV})$ & $0.035 / 2.1$ \\
Length & $L(\mathrm{~m})$ & 4.2 \\
Radio frequency & $f(\mathrm{MHz})$ & 162.5 \\
Normalized transverse & $\epsilon(\pi \mathrm{mm} \mathrm{mrad})$ & 0.3 \\
$\quad$ emittance $(\mathrm{rms})$ & & \\
rms longitudinal emittance & $\epsilon_{z}(\mathrm{keV} \mathrm{deg})$ & 54 \\
Current & $I(\mathrm{~mA})$ & 15 \\
Vane voltage & $V(\mathrm{kV})$ & 65 \\
Min aperture & $a_{\min }(\mathrm{mm})$ & 3.2 \\
Transmission & $\%$ & 99.6 \\
Number of cells & & 192 \\
\hline \hline
\end{tabular}

Figure 9 shows the simulated RFQ acceptance (white area inside the black dots) versus the measured emittance (colored area) at the entrance of the RFQ accelerator. The RFQ has been designed with a large acceptance to comfortably accommodate the injected beam from the proton source.

By measuring the beam energy spectrum at different RFQ input powers, the threshold of the RFQ input power for a good quality beam can be found. From Fig. 10, the working input power of the RFQ should be above $93.6 \mathrm{~kW}$. It corresponds to a designed RFQ intervane voltage of $65 \mathrm{kV}$, which was measured using the x-ray spectrum

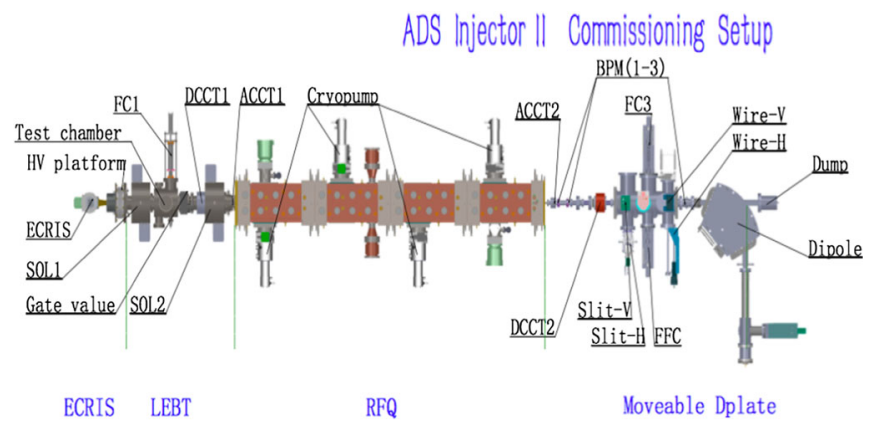

FIG. 8. Commissioning layout of the RFQ.

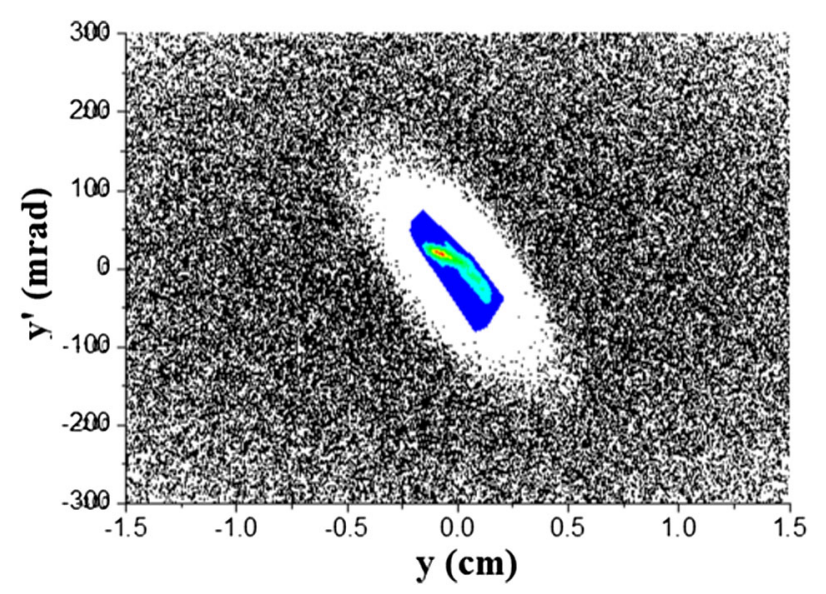

FIG. 9. RFQ acceptance. 


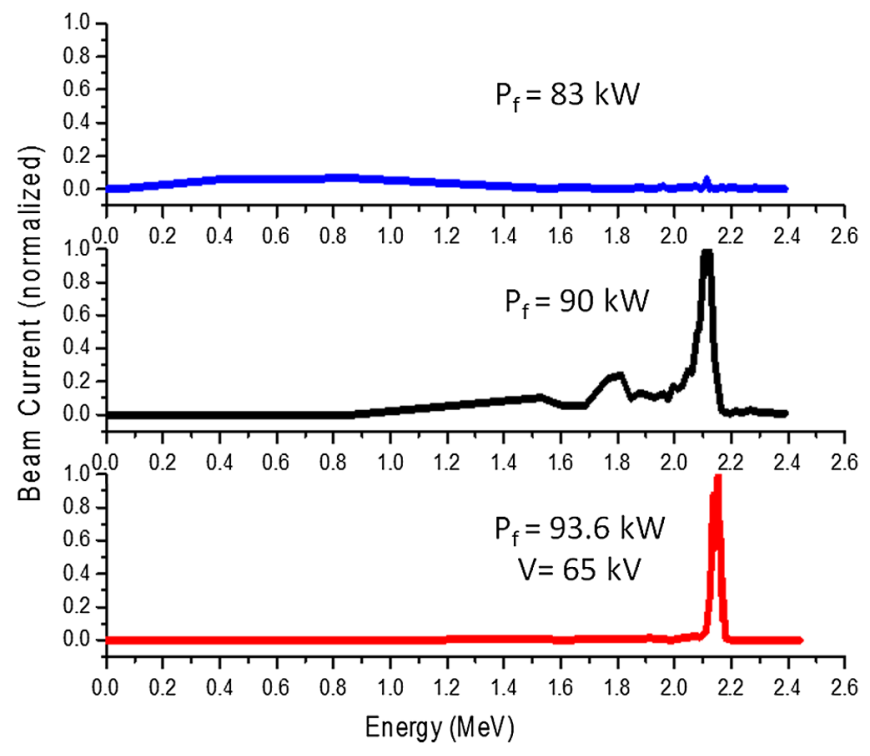

FIG. 10. RFQ beam current versus energy at different input powers.

method. But for normal operation, the RFQ power was conveniently set at $100 \mathrm{~kW}$ by virtue of a more stable $\mathrm{rf}$ power amplifier at the pulsed beam mode.

By utilizing the moving table D-plate beam diagnostic system, the beam energy spectrum from the RFQ can be measured by the magnet as shown in Fig. 11 at a RFQ input power of $93.6 \mathrm{~kW}$. The obtained FWHM energy spread is $1.9 \%$ with a center energy of $2.135 \pm 0.026 \mathrm{MeV}$, which agrees with the TOF measurement of $2.165 \pm 0.005 \mathrm{MeV}$ between BPM1 and BPM3. The measurement shows that the RFQ fellows the design expectation, and the test operation at $10 \mathrm{~mA} \mathrm{CW}$ proton beam ran successfully until the ECR tripped on June 30, 2014 [18]. Also, the measured beam transmission efficient is high to $96.9 \%$ at $10.5 \mathrm{~mA}$.

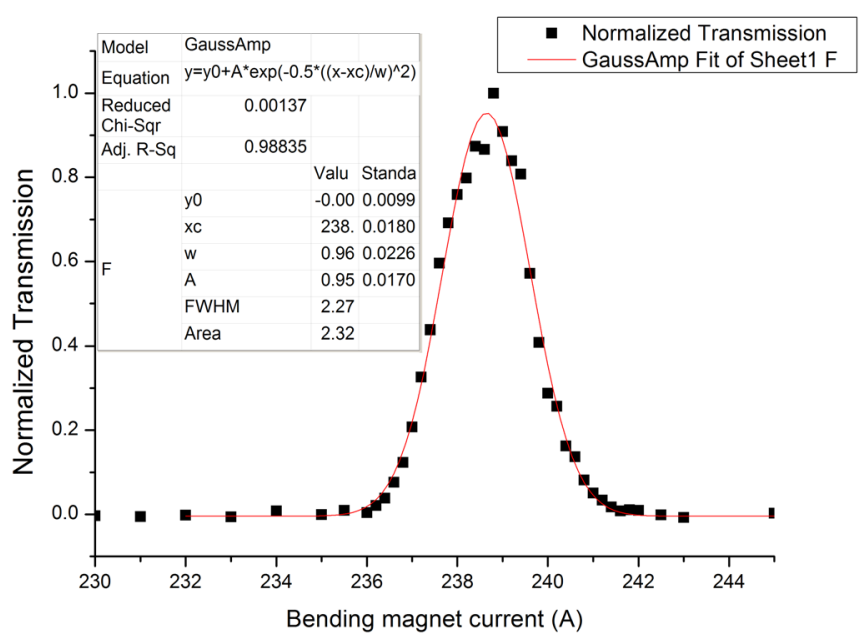

FIG. 11. Beam energy spread of the RFQ.

\section{The MEBT and the test cryomodule}

The MEBT is used to match a beam between the RFQ and superconducting accelerating section in both transversal and longitudinal phase space. Usually, there are a lot of beam diagnostic devices and beam collimation devices, since it is the only room temperature section [19] before the beam goes into the downstream superconducting acceleration section. The layout of the MEBT is shown in Fig. 12. There are seven combine function quads with an orbit correction coil integrated inside each quad. BPM1-4 are installed coaxially inside quads Q1, Q4, Q5, and Q7. There are also slits and scanning wires in both the horizontal and vertical directions for the beam emittance measurement. The two bunching cavities modulate the beam further to match the beam longitudinally into the rf buckets of the downstream superconducting acceleration section.

Since there is no previous experience of building and operating a superconducting accelerator in our institute, a test cryomodule (TCM) with a superconducting HWR cavity and two superconducting solenoids was built for a trial run $[20,21]$. The whole objective of this task was to evaluate the cryogenic system, the superconducting cavity, and the superconducting solenoids. There was a BPM before the cavity and after the first solenoid to demonstrate its reliability. Because the MEBT and the TCM were installed at the same time, the MEBT commissioning was done with the superconducting cavity detuned. Thereafter, the whole system was commissioned. The layout of the MEBT and TCM1 system is shown in Fig. 13. To verify that the MEBT is functioning as designed properly, various beam studies were carried out and compared with offline simulations. Figure 14 shows one example of the emittance measurement compared with the beam simulation results. The right side graphs show the simulated emittance at both the vertical and horizontal directions, while the graphs on the left side show the corresponding measurement ones. They agree very well with each other. Moreover, they also agree with the emittance measurements done using BPM [22]. In order to understand the overall BPM offsets with respect to the center of the quads which the beam orbit is aligned to,

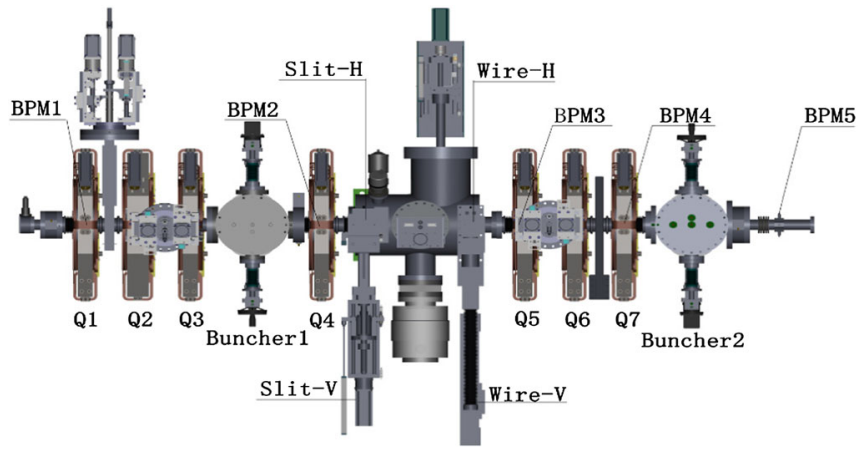

FIG. 12. The layout of the MEBT and beam diagnostics devices. 


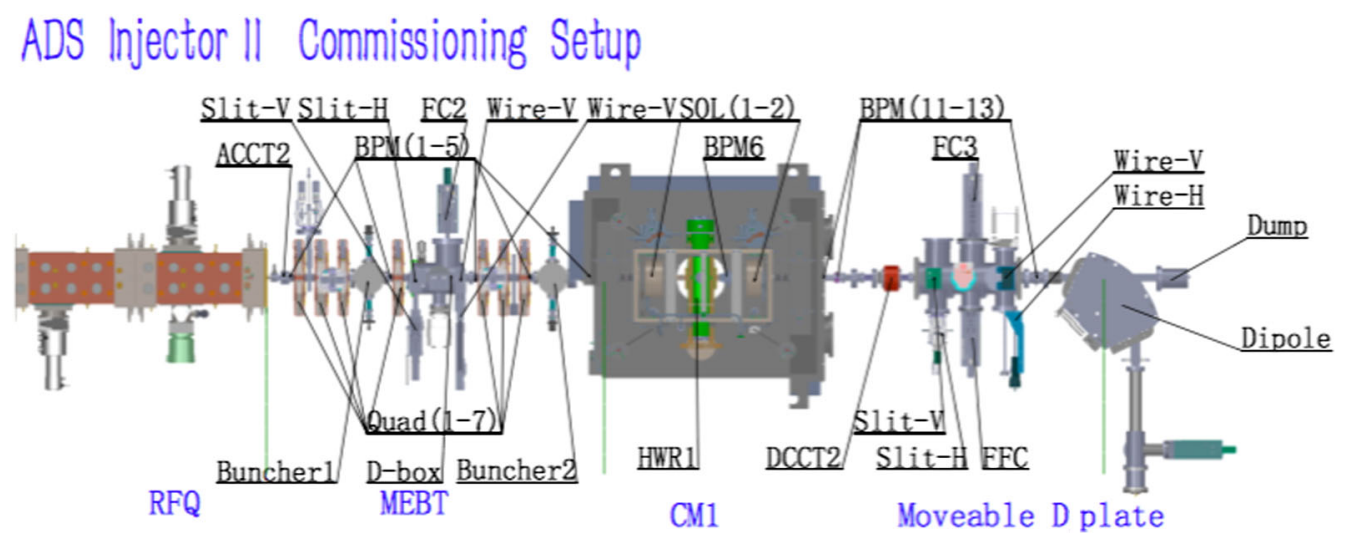

FIG. 13. Layout of the C-ADS injector scheme II with TCM1.
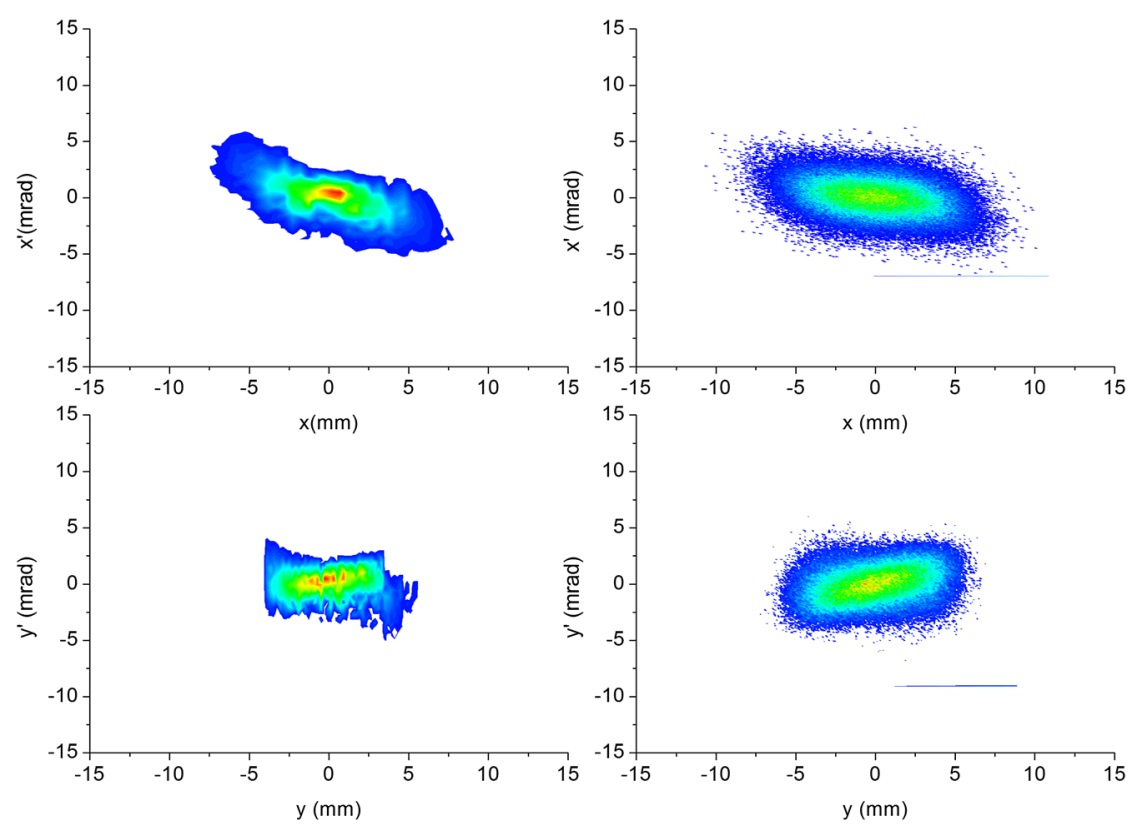

FIG. 14. Emittance simulation and measurements at the MEBT.

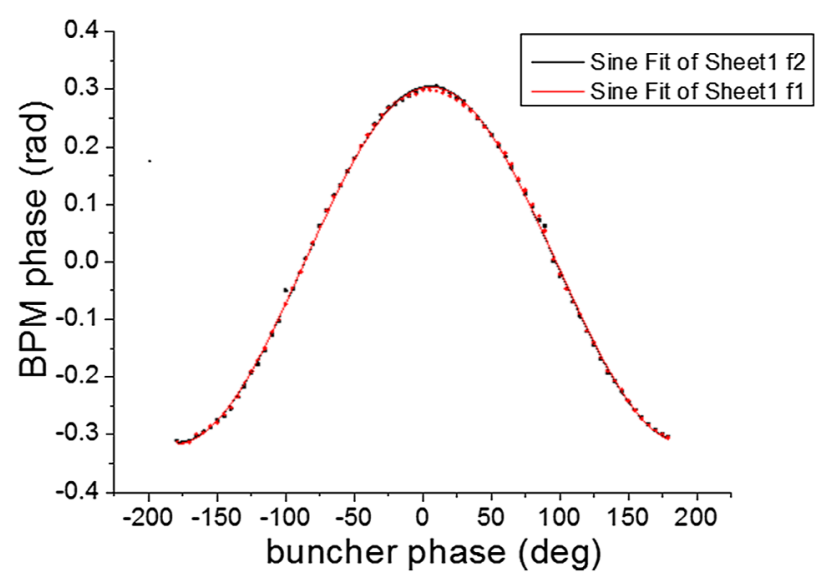

FIG. 15. Phase scan of the buncher. beam-based alignments were performed and the BPM offset values were calibrated [23]. Moreover, the phase scanning and acceleration voltage calibrations of the $\mathrm{rf}$ cavities have been carried out routinely by measuring the TOF of the beam between corresponding BPMs [6]. As an example, the phase scanning of the buncher cavity is shown in Fig. 15. From the scanning result, the voltage was calibrated at $119.8 \mathrm{kV}$, and the phase was set appropriately as well.

By February 13, 2015, an $11 \mathrm{~mA} \mathrm{CW}$ beam acceleration was successfully established and running for about $1 \mathrm{~h}$ at $2.5 \mathrm{MeV}$ with the TCM.

\section{The new cryomodule (CM6)}

With established experience and confidence, the TCM has been replaced by the new CM6, which is shown in 


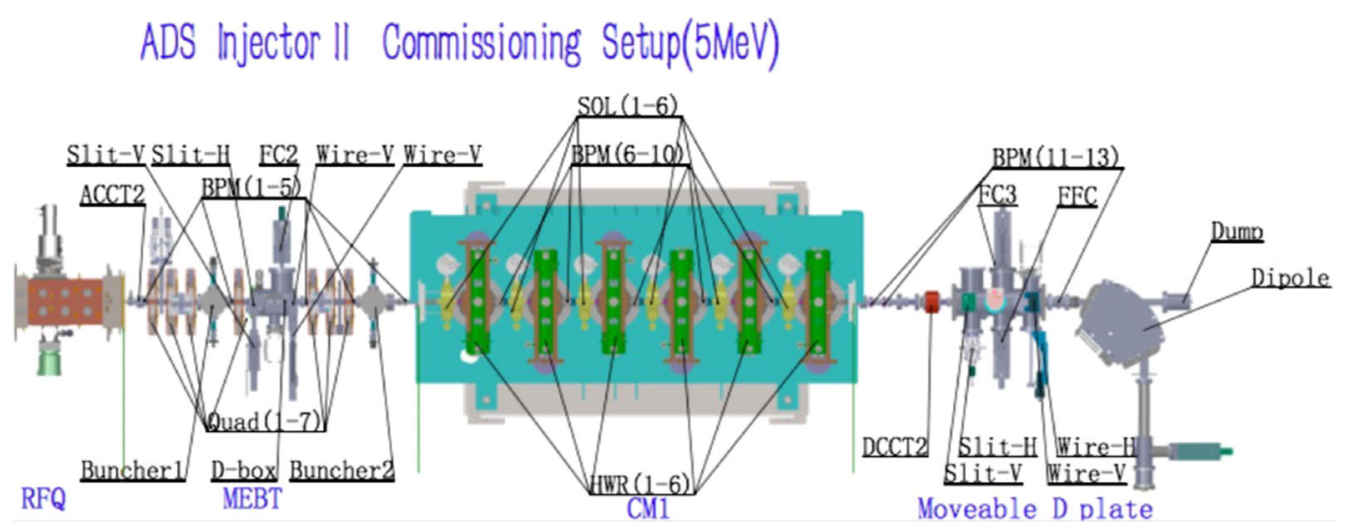

FIG. 16. Layout of the C-ADS injector scheme II with CM6.

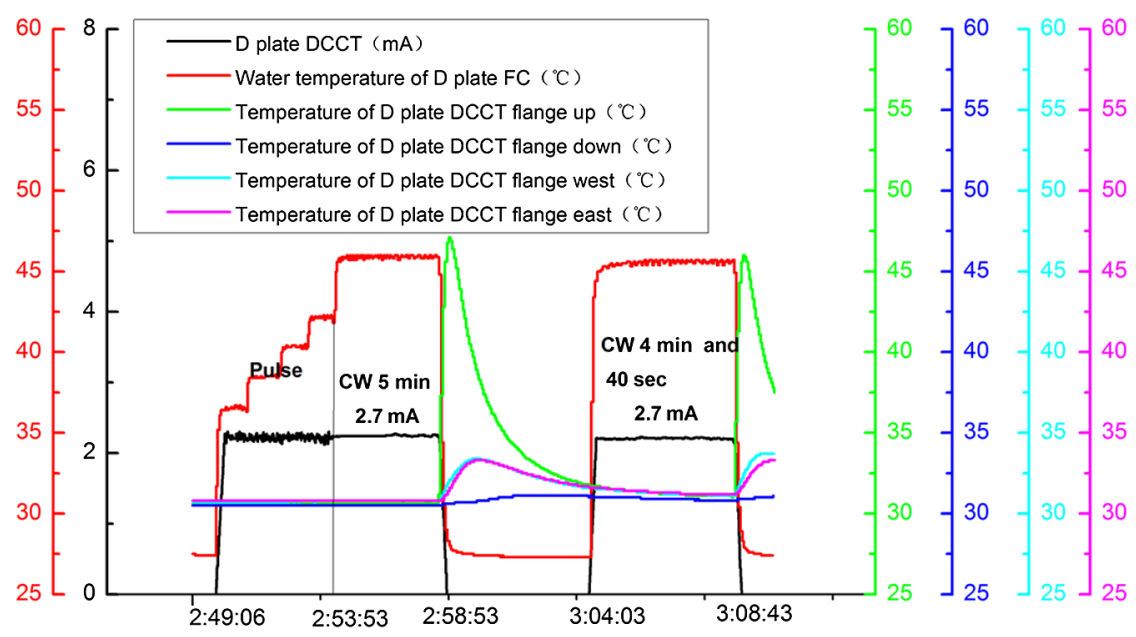

FIG. 17. Two times of CW beam operation at $2.7 \mathrm{~mA}$.

Fig. 16. Inside the CM6, there are six HWR superconducting cavities, six superconducting solenoids, and six BPMs $[20,21]$. The measured beam transmission efficiency is almost $100 \%$ with a final beam energy at $5.3 \mathrm{MeV}$.

After the phase scanning experiments were finished and the acceleration voltage was calibrated for all six HWR cavities, the operating voltages and the phases of the six cavities were carefully set in order with the matching solenoids. The operation of the $\mathrm{CW}$ beam testing was carried out on June 24, 2015, whereas the beam current was intentionally limited at $2.7 \mathrm{~mA}$ using a tentative employment of a Faraday cup as the final beam dump. The history record of the test is shown in Fig. 17. The black line shows a total current of $2.7 \mathrm{~mA}$ at $5.3 \mathrm{MeV}$. The red curve is the temperature of the cooling water for the Faraday cup. The other colored curves are the temperatures measured at different positions of the flange of the DCCT. The operation started with the pulsed beam. Then the beam was transited to $\mathrm{CW}$ mode by adjusting the beam duty factor step by step. The total CW running time lasted for about 10 min before it was shut down by the machine protection system, which was caused by beam loss, quench, and the vacuum leak at the end vacuum valve. The accelerator was shut down because of the new upgrade following the accident. By January 5, 2016, the CW stable operation reached $200 \mathrm{~min}$ at $4 \mathrm{MeV}$ with a $2.7 \mathrm{~mA}$ current.

\section{SUMMARY}

The C-ADS injector II demo accelerator has been under construction, and beam commissioning reached its design goals at each stage. Great experiences have been gained by our young project team during the whole process. The CW acceleration of a proton beam has been carried out successfully. The next phase is aimed to improve the overall reliability and install more accelerating sections. Eventually, the target is to extend the beam energy to $25 \mathrm{MeV}$ at this facility for the designed full prototype linac soon.

\section{ACKNOWLEDGMENTS}

The authors express their great appreciation for the extraordinary dedication and the brilliance of the scientists, engineers, and technicians to keep this project progressing fast and smoothly. The authors also thank our fellow 
colleagues all over the world for all the advice and discussions regarding the machine design and beam commissioning. The work is supported by the National Natural Science Foundation of China (Grants No. 91426303 and No. 11525523) and by the Chinese Academy of Sciences Strategic Priority Research Program-Future Advanced Nuclear Fission Energy (Accelerator-Driven Sub-critical System).

[1] H. At Abderrahimh et al., Reports No. FERMILAB-FN0907-DI, No. LA-UR-10-06754.

[2] F. Carminati, Report No. CERN/AT/93-47(ET), 1993.

[3] C. D. Bowman, Accelerator-driven systems for nuclear waste transmutation, Annu. Rev. Nucl. Part. Sci. 48, 505 (1998).

[4] W.-1. Zhan and H. Xu, Advanced fission energy programADS transmutation system, Bull. Chin. Acad. Sci. 3, 375 (2012).

[5] Z.-j. Wang et al., The design simulation of the superconducting section in the ADS injector, Chin. Phys. C 36, 256 (2012).

[6] H. Jia, Ph.D. dissertation, University of Chinese Academy of Sciences, 2015.

[7] X. Wu, E. Tanke, Q. Zhao, Z.-j. Wang, H. Jia, Y. He, and H. Zhao, in Proceedings of the 4th International Particle Accelerator Conference, IPAC-2013, Shanghai, China, 2013 (JACoW, Shanghai, China, 2013).

[8] D. Uriot and N. Pichoff, in Proceedings of IPAC 2015, Richmond, Virginia, USA (JACoW, Virginia, 2015), MOPWA008.

[9] V. N. Aseev et al., in Proceedings of the 21st Particle Accelerator Conference, Knoxville, TN, 2005 (IEEE, Piscataway, NJ, 2005), pp. 2053-2055.

[10] K. R. Crandall and T. P. Wangler, PARMTEQ-A beamdynamics code fo the RFQ linear accelerator, AIP Conf. Proc. 177, 22 (1988).
[11] Y. K. and Batygin, in Proceedings of the 3rd European Particle Accelerator Conference, Berlin, Germany, 1992 (JaCow, Berlin, 1992), p. 822.

[12] S. Henderson et al., The Spallation Neutron Source accelerator system design, Nucl. Instrum. Methods Phys. Res., Sect. A 763, 610 (2014).

[13] D. Berkovits et al., in Proceedings of LINAC 2012, Tel-Aviv, Israel (JaCow, Tel Aviv, 2012), pp. 100-104.

[14] Z.-j. Wang et al., in Proceedings of LINAC 2012, Tel-Aviv, Israel (JaCow, Tel Aviv, 2012).

[15] Q. Wu, Z. M. Zhang, L. T. Sun, Y. Yang, H. Y. Ma, Y. Cao, X. Z. Zhang, and H. W. Zhao, A $2.45 \mathrm{GHz}$ intense proton source and low energy beam transport system for China Initiative Accelerator Driven Sub-Critical reactor system, Rev. Sci. Instrum. 85, 02 A703 (2014).

[16] Z. Zhouli et al., Design and beam test of a high intensity continuous wave RFQ accelerator, Nucl. Instrum. Methods Phys. Res., Sect. A 763, 383 (2014).

[17] L. Chao, Z. Zhi-Lei, Q. Xin, X. Xian-Bo, H. Yuan, and Y. Lei, Beam dynamics study of RFQ for CADS with a 3D space-charge-effect, Chin. Phys. C 38, 037005 (2014).

[18] Yuan He, in Proceedings of HIAT 2015, Yokohama, Japan (JaCow, Yokohama, 2015).

[19] H. Jia, Y. Yuan, M. Song, Y. He, C. Luo, X. Zhang, and S. Huang, in Proceedings of HB 2012 (JaCow, Beijing, 2015).

[20] Z. Cong, H. Shou-Bo, W. Ruo-Xu, Z. Sheng-Hu, H. Yuan, and Z. Hong-Wei, Structural analysis of quarterwave resonators in IMP, Chin. Phys. C 37, 107002 (2013).

[21] W. Yue and Y. He, in Proceedings of SRF 2013, Paris, France (JaCow, Paris, 2013).

[22] Z.J. Wang et al., Noninterceptive transverse emittance measurements using BPM for Chinese ADS R\&D project, Nucl. Instrum. Methods Phys. Res., Sect. A 816, 171 (2016).

[23] C. Weilong et al., Chin. Phys. C 40, 077003 (2016). 